\title{
Tangential 2-D Edge Imaging for GPI and Edge/Impurity Modeling
}

Final Report for the period June 15, 2004 - June 14, 2010

Prepared by: Dr. Ricardo Maqueda \& Dr. Fred M. Levinton

Nova Photonics, Inc.

Princeton, NJ 08540

Prepared for

THE U.S. DEPARTMENT OF ENERGY

AWARD NO. DE-FG02-04ER54767 


\section{Executive Summary}

Nova Photonics, Inc. has a collaborative effort at the National Spherical Torus Experiment (NSTX). This collaboration, based on fast imaging of visible phenomena, has provided key insights on edge turbulence, intermittency, and edge phenomena such as edge localized modes (ELMs) and multi-faceted axisymmetric radiation from the edge (MARFE). Studies have been performed in all these areas. The edge turbulence/intermittency studies make use of the Gas Puff Imaging diagnostic developed by the Principal Investigator (Ricardo Maqueda) together with colleagues from PPPL. This effort is part of the International Tokamak Physics Activity (ITPA) edge, scrape-off layer and divertor group joint activity (DSOL15: Inter-machine comparison of blob characteristics). The edge turbulence/blob study has been extended from the current location near the midplane of the device to the lower divertor region of NSTX. The goal of this effort was to study turbulenceborn blobs in the vicinity

of the X-point region and their circuit closure on divertor sheaths or high density regions in the divertor. In the area of ELMs and MARFEs we have studied and characterized the mode structure and evolution of the ELM types observed in NSTX, as well as the study of the observed interaction between MARFEs and ELMs. This interaction could have substantial implications for future devices where radiative divertor regions are required to maintain detachment from the divertor plasma facing components. 


\section{Abstract}

Nova Photonics, Inc. has a collaborative effort at the National Spherical Torus Experiment (NSTX). This collaboration, based on fast imaging of visible phenomena, has provided key insights on edge turbulence, intermittency, and edge phenomena such as edge localized modes (ELMs) and multi-faceted axisymmetric radiation from the edge (MARFE).

\section{Results}

Nova Photonics, Inc. and the principal investigator of this project, Ricardo Maqueda, have been involved in fast-imaging of edge phenomena in NSTX since July 2004. This collaboration has involved the development of the Gas Puff Imaging diagnostic (or GPI) and the study of edge turbulence facilitated by this diagnostic. This research has led to the identification intermittency seen on the lower divertor target plates and their correlation with midplane intermittency (blobs). A summary of the results so far obtained are summarized further below in this Section.

During recent years Dr. Maqueda has also been involved in the study of the fine structure of ELMs, as well as in the study of the interaction of ELMs with main chamber MARFEs. With the use of a fast-framing camera such interaction was first documented in NSTX. Among other things, it was observed that a toroidally localized MARFE precursor results from the partial burn-through of a pre-existent MARFE by energy liberated from the closed field line region during the ELM crash. This precursor may subsequently expand into a toroidally symmetric MARFE.

The study of the fine structure of ELMs has shown that the filamentation observed in 
other experiments, which is also observed in NSTX, is followed by a relatively longer period of secondary filaments. These filaments have all the characteristics of the turbulent born blobs observed during Ohmic, L-mode and inter-ELM periods of H-mode discharges. The most notable difference between inter-ELM period intermittency and secondary filamentation during the ELM event is the level of activity, resembling more an L-mode discharge than the inter- ELM period. A summary of the results obtained in this area of research are also summarized further below in this Section.

In addition to the study of edge turbulence, blobs, ELM's and MARFE's, the use of fastframing camera has been instrumental in the development of Transient Coaxial Helicity Injection as well as the study of dust in NSTX. The main aspects of the recent progress in these areas are described below. The results presented are intended only to illustrate the physics issues that are being studied.

Fast moving striations have been observed in the lower divertor target plates. Due to the low ionization energy of neutral lithium, the LiI light emission originates close to the divertor target plates and corresponds to footprints created by the interaction of localized higher density and temperature plasma with the target plates. The striations move toroidally as time progresses. An analysis of the characteristics of these striations as function of the major radius $\mathrm{R}$ measured along the divertor target plates has been completed. The root-meansquare fluctuation level, skewness and kurtosis are similar to those seen near the midplane with the GPI diagnostic. Furthermore, the movement of these footprints, toroidally in the direction of co-current are consistent with the predominant movement of the intermittent events (blobs) that have been observed near the midplane, where they move radially out and poloidally down (ion diamagnetic drift direction). Making use of the two fast-framing digital cameras that Nova Photonics has fielded on NSTX the intermittency observed on the lower divertor target plates can be correlated with the intermittent blobs seen with the midplane 
GPI diagnostic (located just above the outer midplane).

The results summarized above provide then a good indication that the midplane scrape-off layer is well connected to the divertor target plates, at least in its intermittent features. It should be noted also that the divertor emission becomes more Gaussian closer to the outer strike point. These features are being investigated as part of the current grant and the research will be continued as part of the grant continuation requested here.

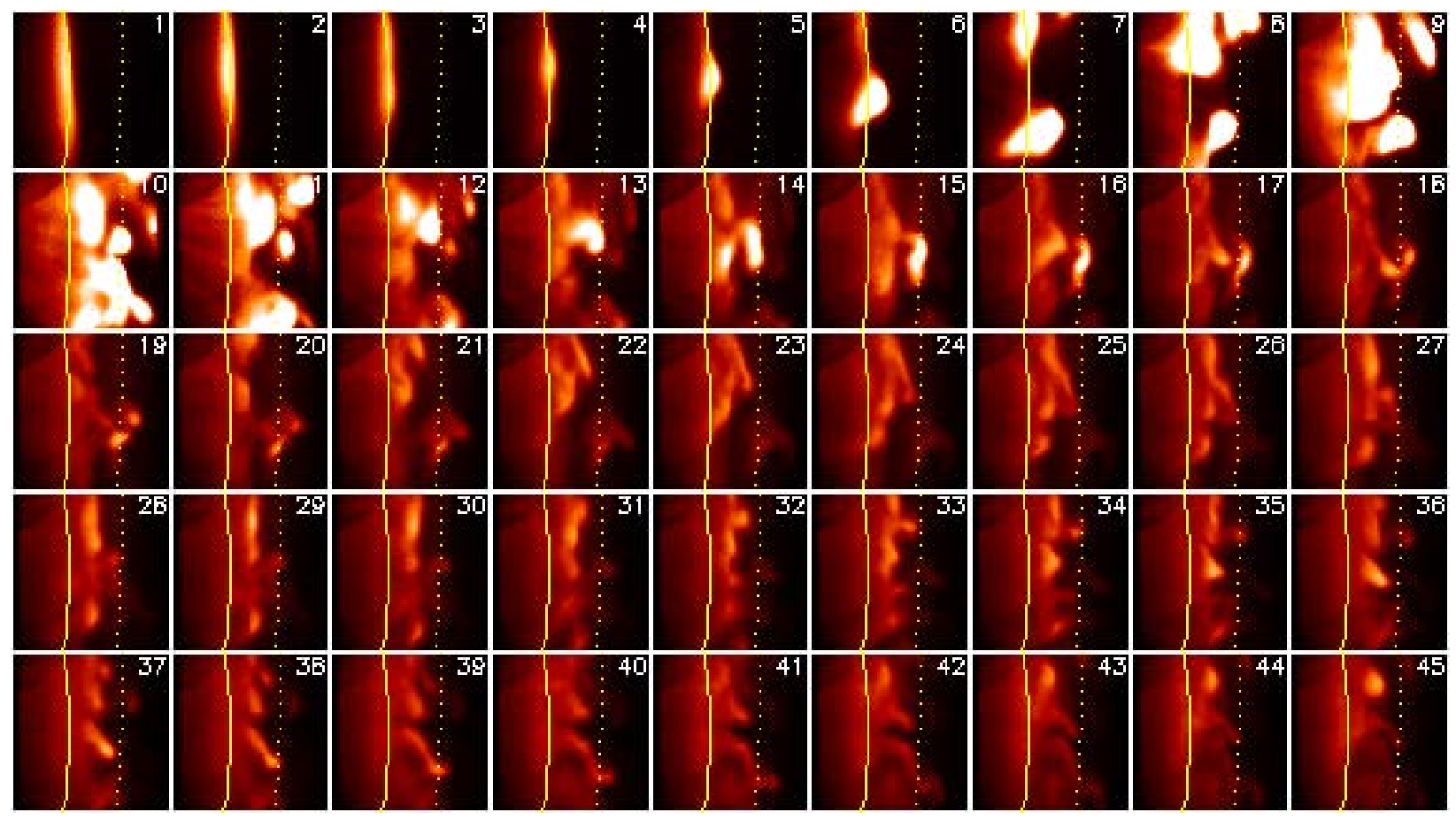

Figure 1: Gas puff imaging during Type III ELM. While primary ELM filaments are seen during the early stages of the ELM event (frames 5-11), secondary filaments due to the ELM can be seen on later frames. (Shot 124667, $262.543 \mathrm{~ms}$ to $262.905 \mathrm{~ms}$ ).

The GPI diagnostic has been used to study the fine structure of ELMs present in NSTX during H-mode discharges. One example obtained during a Type III ELM is shown in Figure 1. In this figure it is seen how a filament (frame \#6) evolves from the unperturbed Hmode edge (frame \#2), first as a higher density and/or temperature region (i.e., brighter $\mathrm{D}_{\alpha}$ 
emission) on the edge that then moves radially outwards and poloidally in the ion diamagnetic drift direction (downwards in this case) as seen in Figure 1, frames \#5-\#9, in a similar way to that reported from other experiments. Generally, due to the break-up of the primary filaments, the edge continues to develop into further filaments and hence an increased toroidal quasi-mode number, resulting in a fine structured filament pattern (frame \#9). The edge, perturbed by the ELM, is then subject to increased turbulence and blob generation as seen in the subsequent frames of Figure 1 resulting in an even higher toroidal quasi-mode number since these turbulent blobs are also aligned with the local magnetic field. These later structures are the secondary filaments corresponding to this Type III ELM event. Secondary filament activity decays in $\sim 200 \mu$ s after the first onset of the ELM, recovering the typical H-mode edge seen in frames \#1-\#2.

\section{Summary of Accomplishments}

During the current grant (period June 2004 through June 2010) the accomplishments include:

- Implementation of new fast-framing camera (Phantom 7.1 from Vision Research) on NSTX, with Labview control software to synchronize data acquisition with NSTXs shot cycle.

- Development of software for analysis of intermittent (blob) structures, including automated tracking of blobs.

- Study of scaling of intermittent activity during H-modes in NSTX.

- Study of ELM structure in the divertor, including the propagation of ELM signatures along the inner leg of the divertor up to the $\mathrm{X}$-point and its implication on past 
inferences of the birth location of the ELM.

- Interpretation of the small (Type V) ELMs in NSTX as a long-lived (up to $1 \mathrm{~ms}$ ) rotating perturbation of the magnetic flux surfaces without the need for individual filaments breaking off.

- First observation of the interaction between ELMs and main chamber MARFEs as well as the presence of toroidally localized "MARFE precursors" that lead into the creation of the traditional MARFE.

- Implementation of second fast-framing camera (Phantom 7.3 from Vision Research) on NSTX. Integration with Labview control software allowing the control of 2 or more Vision Research cameras with the same Labview Virtual Instrument, while having the data acquisition cycle of the cameras synchronized with NSTXs shot cycle.

- Hardware developed to synchronize the frame capture of the two Phantom cameras to better than $0.25 \mu \mathrm{s}$, without the introduction of ground loops.

- Identification of intermittency on the lower divertor target plates and their correlation to midplane intermittency.

- Identification of secondary filaments during the ELM event and their characteristic. These secondary filaments correspond to turbulence produced blobs which are born from perturbed edge profiles as a consequence of the ELM.

\section{$5 \quad$ List of Invited Talks and Publications}

\section{INVITED TALKS}

"ELM filament structure in the National Spherical Torus Experiment", 50th Annual Meet- 
ing of the Division of Plasma Physics of the American Physical Society, Nov. 17-21, 2008, Dallas, Texas, USA.

\section{REFEREED PUBLICATIONS (First author)}

"Intermittent divertor filaments in the National Spherical Torus Experiment and their relation to midplane blobs", R. J. Maqueda and the NSTX Team, to be submitted to Nucl. Fusion.

"Secondary ELM filaments in NSTX", R. J. Maqueda, R. Maingi, and J.-W. Ahn, J. Nucl. Mater. 390-391, p. 843, June 2009.

"Primary Edge Localized Mode Filament Structure in the National Spherical Torus Experiment", R. J. Maqueda and R. Maingi, Phys. Plasmas 16, Art. No. 056117, May 2009.

"Structure of MARFEs and ELMs in NSTX", R. J. Maqueda, R. Maingi, K. Tritz, K. C.

Lee, C. E. Bush, E. D. Fredrickson, J. E. Menard, A. L. Roquemore, S. A. Sabbagh and S. J. Zweben, J. Nucl. Mater. 363-365, p. 1000, June 2007. 